\title{
Complex Oscillation of Higher-Order Linear Differential Equations with Coefficients Being Lacunary Series of Finite Iterated Order
}

\author{
Jin Tu, ${ }^{1}$ Hong-Yan $\mathrm{Xu}^{2}{ }^{2}$ Hua-ming Liu, ${ }^{3}$ and Yong Liu ${ }^{4,5}$ \\ ${ }^{1}$ College of Mathematics and Information Science, Jiangxi Normal University, Nanchang 330022, China \\ ${ }^{2}$ Department of Informatics and Engineering, Jingdezhen Ceramic Institute, Jingdezhen, Jiangxi 333403, China \\ ${ }^{3}$ School of Sciences, Jiangxi Agricultural University, Nanchang 330045, China \\ ${ }^{4}$ Department of Mathematics, Shaoxing College of Arts and Sciences, Shaoxing, Zhejiang 312000, China \\ ${ }^{5}$ Department of Physics and Mathematics, University of Eastern Finland, P.O. Box 111, 80101 Joensuu, Finland
}

Correspondence should be addressed to Jin Tu; tujin2008@sina.com

Received 18 January 2013; Accepted 12 April 2013

Academic Editor: Micah Osilike

Copyright ( 2013 Jin Tu et al. This is an open access article distributed under the Creative Commons Attribution License, which permits unrestricted use, distribution, and reproduction in any medium, provided the original work is properly cited.

\begin{abstract}
The authors introduce the lacunary series of finite iterated order and use them to investigate the growth of solutions of higher-order linear differential equations with entire coefficients of finite iterated order and obtain some results which improve and extend some previous results of Belaidi, 2006, Cao and Yi, 2007, Kinnunen, 1998, Laine and Wu, 2000, Tu and Chen, 2009, Tu and Deng, 2008, Tu and Deng, 2010, Tu and Liu, 2009, and Tu and Long, 2009.
\end{abstract}

\section{Definitions and Notations}

In this paper, we assume that readers are familiar with the fundamental results and standard notations of the Nevanlinna theory of meromorphic functions (see [1-3]). In order to describe the growth of order of entire functions or meromorphic functions more precisely, we first introduce some notations about finite iterated order. Let us define inductively, for $r \in(0,+\infty), \exp _{1} r=e^{r}$ and $\exp _{i+1} r=\exp \left(\exp _{i} r\right), i \in$ $\mathbb{N}$. For all sufficiently large $r$, we define $\log _{1} r=\log r$ and $\log _{i+1} r=\log \left(\log _{i} r\right), i \in \mathbb{N}$. We also denote $\exp _{0} r=r=\log _{0} r$ and $\exp _{-1} r=\log _{1} r$. Moreover, we denote the logarithmic measure of a set $E \subset(0,+\infty)$ by $m_{l} E=\int_{E} d t / t$, and the upper logarithmic density of $E \subset(0,+\infty)$ is defined by

$$
\overline{\log \operatorname{dens}} E=\varlimsup_{r \rightarrow \infty} \frac{m_{l}(E \bigcap[1, r])}{\log r} .
$$

Throughout this paper, we use $p$ to denote a positive integer. In the following, we recall some definitions of entire functions or meromorphic functions of finite iterated order (see [410]).
Definition 1. The $p$-iterated order of a meromorphic function $f(z)$ is defined by

$$
\sigma_{p}(f)=\varlimsup_{r \rightarrow \infty} \frac{\log _{p} T(r, f)}{\log r} .
$$

Remark 2. If $f(z)$ is an entire function, then the $p$-iterated order of $f(z)$ is defined by

$$
\sigma_{p}(f)=\varlimsup_{r \rightarrow \infty} \frac{\log _{p} T(r, f)}{\log r}=\varlimsup_{r \rightarrow \infty} \frac{\log _{p+1} M(r, f)}{\log r} .
$$

If $p=1$, the classical growth of order of $f(z)$ is defined by

$$
\sigma(f)=\varlimsup_{r \rightarrow \infty} \frac{\log T(r, f)}{\log r}=\varlimsup_{r \rightarrow \infty} \frac{\log _{2} M(r, f)}{\log r} .
$$

If $p=2$, the hyperorder of $f(z)$ is defined by

$$
\sigma_{2}(f)=\varlimsup_{r \rightarrow \infty} \frac{\log _{2} T(r, f)}{\log r}=\varlimsup_{r \rightarrow \infty} \frac{\log _{3} M(r, f)}{\log r} .
$$


Definition 3. If $f(z)$ is an entire function with $0<\sigma_{p}(f)=$ $\sigma<\infty$, then the $p$-iterated type of $f(z)$ is defined by

$$
\tau_{p}(f)=\varlimsup_{r \rightarrow \infty} \frac{\log _{p} M(r, f)}{r^{\sigma}} .
$$

Definition 4. The $p$-iterated lower order of an entire function $f(z)$ is defined by

$$
\mu_{p}(f)=\lim _{r \rightarrow \infty} \frac{\log _{p} T(r, f)}{\log r}=\lim _{r \rightarrow \infty} \frac{\log _{p+1} M(r, f)}{\log r} .
$$

Definition 5. The finiteness degree of the iterated order of a meromorphic function $f(z)$ is defined by

$i(f)$

$$
= \begin{cases}0 \quad & \text { for } f(z) \text { rational, } \\ \min \left\{p \in \mathbb{N}: \sigma_{p}(f)<\infty\right\} & \\ & \text { for } f \text { transcendental for which some } \\ & p \in \mathbb{N} \text { with } \sigma_{p}(f)<\infty \text { exists, } \\ \infty \quad & \text { or } f(z) \text { with } \sigma_{p}(f)=\infty \forall p \in \mathbb{N} .\end{cases}
$$

Definition 6. The $p$-iterated exponent of convergence of $a$ point of a meromorphic function $f(z)$ is defined by

$$
\lambda_{p}(f, a)=\varlimsup_{r \rightarrow \infty} \frac{\log _{p} n(r, a)}{\log r}=\varlimsup_{r \rightarrow \infty} \frac{\log _{p} N(r, a)}{\log r} .
$$

If $a=0$, the $p$-iterated exponent of convergence of zero-sequence and distinct zero-sequence of a meromorphic function $f(z)$ are defined, respectively, by

$$
\begin{aligned}
& \lambda_{p}(f)=\varlimsup_{r \rightarrow \infty} \frac{\log _{p} n(r, 1 / f)}{\log r}=\varlimsup_{r \rightarrow \infty} \frac{\log _{p} N(r, 1 / f)}{\log r}, \\
& \bar{\lambda}_{p}(f)=\varlimsup_{r \rightarrow \infty} \frac{\log _{p} \bar{n}(r, 1 / f)}{\log r}=\varlimsup_{r \rightarrow \infty} \frac{\log _{p} \bar{N}(r, 1 / f)}{\log r} .
\end{aligned}
$$

The $p$-iterated lower exponent of convergence of zerosequence and distinct zero-sequence of a meromorphic function $f(z)$ are defined, respectively, by

$$
\begin{gathered}
\underline{\lambda}_{p}(f)=\lim _{r \rightarrow \infty} \frac{\log _{p} \bar{n}(r, 1 / f)}{\log r}=\lim _{r \rightarrow \infty} \frac{\log _{p} \bar{N}(r, 1 / f)}{\log r}, \\
\underline{\bar{\lambda}}_{p}(f)=\lim _{r \rightarrow \infty} \frac{\log _{p} \bar{n}(r, 1 / f)}{\log r}=\lim _{r \rightarrow \infty} \frac{\log _{p} \bar{N}(r, 1 / f)}{\log r} .
\end{gathered}
$$

\section{Introductions and Main Results}

In the past ten years, many authors have investigated the complex oscillation properties of the higher-order linear differential equations

$$
\begin{gathered}
f^{(k)}+A_{k-1}(z) f^{(k-1)}+\cdots+A_{0}(z) f=0, \\
f^{(k)}+A_{k-1}(z) f^{(k-1)}+\cdots+A_{0}(z) f=F(z)
\end{gathered}
$$

with $A_{j}(z)(j=0, \ldots, k-1), F(z)$ being entire functions or meromorphic functions of fast growing (e.g., see [4-12]), and obtained the following results.
Theorem A (see [8]). Let $A_{0}(z), \ldots, A_{k-1}(z)$ be entire functions, if $i\left(A_{j}\right) \leq p(j=0, \ldots, k-1)$, then $i(f) \leq p+1$ and $\sigma_{p+1}(f) \leq \max \left\{\sigma_{p}\left(A_{j}\right), j=0, \ldots, k-1\right\}$ hold for all solutions of (12).

Theorem B (see [8]). Let $A_{0}(z), \ldots, A_{k-1}(z)$ be entire functions and let $i\left(A_{0}\right)=p$. If $i\left(A_{j}\right)<p$ or $\sigma_{p}\left(A_{j}\right)<\sigma_{p}\left(A_{0}\right)$ for all $j=1, \ldots, k-1$, then $i(f)=p+1$ and $\sigma_{p+1}(f)=\sigma_{p}\left(A_{0}\right)$ hold for all nontrivial solutions of (12).

Theorem C (see $[4,12])$. Let $A_{0}(z), \ldots, A_{k-1}(z)$ be entire functions and let $i\left(A_{0}\right)=p$. Assume that $\max \left\{\sigma_{p}\left(A_{j}\right): j=\right.$ $1, \ldots, k-1\} \leq \sigma_{p}\left(A_{0}\right)(>0)$ and $\max \left\{\tau_{p}\left(A_{j}\right): \sigma_{p}\left(A_{j}\right)=\right.$ $\left.\sigma_{p}\left(A_{0}\right)\right\}<\tau_{p}\left(A_{0}\right)$. Then, every solution $f(z) \not \equiv 0$ of $(12)$ satisfies $i(f)=p+1$ and $\sigma_{p+1}(f)=\sigma_{p}\left(A_{0}\right)$.

Theorem $\mathrm{D}$ (see [10]). Let $A_{0}(z), \ldots, A_{k-1}(z)$ be entire functions of finite iterated order satisfying $i\left(A_{0}\right)=p, \sigma_{p}\left(A_{0}\right)=$ $\sigma$, and $\varlimsup_{\lim _{r \rightarrow \infty}}\left(\sum_{j=1}^{k-1} m\left(r, A_{j}\right) / m\left(r, A_{0}\right)\right)<1$. Then, every nontrivial solution $f(z)$ of $(12)$ satisfies $\sigma_{p+1}(f)=\sigma_{p}\left(A_{0}\right)=$ $\sigma$.

Theorem E (see [10]). Let $A_{0}(z), \ldots, A_{k-1}(z)$ be entire functions of finite iterated order satisfying $\max \left\{\sigma_{p}\left(A_{j}\right), j \neq 0\right\}<$ $\mu_{p}\left(A_{0}\right)=\sigma_{p}\left(A_{0}\right)$ and $\varlimsup_{\lim _{r \rightarrow \infty}}\left(\sum_{j=1}^{k-1} m\left(r, A_{j}\right) / m\left(r, A_{0}\right)\right)<$ $1(r \notin E)$, where $E$ is a set of $r$ of finite linear measure, then every nontrivial solution $f(z)$ of $(12)$ satisfies $\sigma_{p+1}(f)=$ $\mu_{p}\left(A_{0}\right)=\sigma_{p}\left(A_{0}\right)$.

Theorem F (see [5]). Let $A_{j}(z)(j=0, \ldots, k-1)$ be entire functions of finite iterated order such that there exists one transcendental $A_{d}(0 \leq d \leq k-1)$ satisfying $\sigma_{p}\left(A_{j}\right) \leq$ $\sigma_{p}\left(A_{d}\right)<\infty$ for all $j \neq s$, then (12) has at least one solution $f(z)$ that satisfies $i(f)=p+1$ and $\sigma_{p+1}(f)=\sigma_{p}\left(A_{d}\right)$.

Remark 7. Theorems $\mathrm{B}-\mathrm{E}$ are investigating the growth of solutions of (12) when the coefficients are of finite iterated order and $A_{0}(z)$ grows faster than other coefficients in (12). What can we have if there exists one middle coefficient $A_{d}(z)(1 \leq d \leq k-1)$ such that $A_{d}(z)$ grows faster than other coefficients in (12) or (13)? Many authors have investigated this question when $A_{d}(z)$ is of finite order and obtained many results (e.g., see [13-15]). Here, our question is that under what conditions can we obtain similar results with Theorems B-C if $A_{d}(z)(1 \leq d \leq k-1)$ is of finite iterated order and grows faster than other coefficients in (12) or (13).

In 2009, Tu and Liu make use of the proposition of lacunary power series to investigate the above question in the case $p=1$ and obtain the following result.

Theorem G (see [15]). Let $A_{j}(z)(j=0, \ldots, k-1), F(z)$ be entire functions satisfying $\max \left\{\sigma\left(A_{j}\right), j \neq d, \sigma(F)\right\}<$ $\sigma\left(A_{d}\right)<\infty(1 \leq d \leq k-1)$. Suppose that $A_{d}(z)=\sum_{n=0}^{\infty} c_{\lambda_{n}} z^{\lambda_{n}}$ is an entire function of regular growth such that the sequence of exponents $\left\{\lambda_{n}\right\}$ satisfies Fabry gap condition

$$
\frac{\lambda_{n}}{n} \longrightarrow \infty \quad(n \longrightarrow \infty)
$$


then one has

(i) if $F(z) \equiv 0$, then every transcendental solution $f(z)$ of (13) satisfies $\sigma_{2}(f)=\sigma\left(A_{d}\right)$;

(ii) if $F(z) \neq \equiv$, then every transcendental solution $f(z)$ of (13) satisfies $\bar{\lambda}_{2}(f)=\lambda_{2}(f)=\sigma_{2}(f)=\sigma\left(A_{d}\right)$.

In this paper, we continue our research in this area and obtain the following results.

Theorem 8. Let $A_{j}(z)(j=0, \ldots, k-1), F(z)$ be entire functions of finite iterated order and satisfying $0<$ $\max \left\{\sigma_{p}\left(A_{j}\right), j \neq d\right\} \leq \sigma_{p}\left(A_{d}\right)<\infty$ and $\max \left\{\tau_{p}\left(A_{j}\right):\right.$ $\left.\sigma_{p}\left(A_{j}\right)=\sigma_{p}\left(A_{d}\right)\right\}<\tau_{p}\left(A_{d}\right)(0 \leq d \leq k-1)$. Suppose that $A_{d}(z)=\sum_{n=0}^{\infty} c_{\lambda_{n}} z^{\lambda_{n}}$ is an entire function such that the sequence of exponents $\left\{\lambda_{n}\right\}$ satisfies

$$
\frac{\lambda_{n}}{n}>(\log n)^{2+\eta} \quad(\eta>0, n \in \mathbb{N})
$$

for some $\eta>0$, then one has

(i) if $\sigma_{p}(F)<\sigma_{p}\left(A_{d}\right)$ or $\sigma_{p}(F)=\sigma_{p}\left(A_{d}\right)$ and $\tau_{p}(F)<$ $\tau_{p}\left(A_{d}\right)$, then every transcendental solution $f(z)$ of $(13)$ satisfies $\sigma_{p+1}(f)=\sigma_{p}\left(A_{d}\right)$; furthermore if $F(z) \neq \equiv$, then every transcendental solution $f(z)$ of (13) satisfies $\bar{\lambda}_{p+1}(f)=\lambda_{p+1}(f)=\sigma_{p+1}(f)=\sigma_{p}\left(A_{d}\right) ;$

(ii) if $\sigma_{p}(F)>\sigma_{p}\left(A_{d}\right)$ and $\sigma_{p+1}(F) \leq \sigma_{p}\left(A_{d}\right)$, then all solutions of (13) satisfy $\sigma_{p}(f) \geq \sigma_{p}(F)$ and $\sigma_{p+1}(f) \leq$ $\sigma_{p}\left(A_{d}\right)$;

(iii) if $\sigma_{p+1}(F)>\sigma_{p}\left(A_{d}\right)$, then all solutions of (13) satisfy $\sigma_{p+1}(f)=\sigma_{p+1}(F)$, and $\bar{\lambda}_{p+1}(f)=\lambda_{p+1}(f)=$ $\sigma_{p+1}(F)$ holds for all solutions of (13) with at most one exceptional solution $f_{0}$ satisfying $\lambda_{p+1}\left(f_{0}\right)<\sigma_{p+1}(F)$.

Remark 9. If $f(z)=\sum_{n=0}^{\infty} c_{\lambda_{n}} z^{\lambda_{n}}$ is an entire function of finite order and the sequence of exponents $\left\{\lambda_{n}\right\}$ satisfies (14), then (18) in Lemma 15 holds for $f(z)$, but for entire functions of infinite order, (14) certainly does not imply (18) in Lemma 15 (see [8]); therefore, we need more stringent gap condition (15) which is sufficient and unnecessary for Theorem 8 .

Theorem 10. Let $A_{j}(z)(j=0, \ldots, k-1), F(z)$ be entire functions of finite iterated order satisfying $\max \left\{\sigma_{p}\left(A_{j}\right)\right.$, $\left.j \neq d, \sigma_{p}(F)\right\}<\mu_{p}\left(A_{d}\right)=\sigma_{p}\left(A_{d}\right)=\sigma<\infty(0 \leq d \leq$ $k-1)$. Suppose that $A_{d}(z)=\sum_{n=0}^{\infty} c_{\lambda_{n}} z^{\lambda_{n}}$ is an entire function such that the sequence of exponents $\left\{\lambda_{n}^{n}\right\}$ satisfies gap condition (15), then every transcendental solution $f(z)$ of (13) satisfies $\mu_{p+1}(f)=\sigma_{p+1}(f)=\sigma$. Furthermore if $F(z) \neq \equiv$, then every transcendental solution $f(z)$ of (13) satisfies $\underline{\bar{\lambda}}_{p+1}(f)=$ $\underline{\lambda}_{p+1}(f)=\bar{\lambda}_{p+1}(f)=\lambda_{p+1}(f)=\sigma$.

Theorem 11. Let $A_{j}(z)(j=0, \ldots, k-1), F(z)$ be entire functions satisfying $\max \left\{\sigma_{p}\left(A_{j}\right), j \neq d\right\}<\sigma_{p}\left(A_{d}\right)<\infty(0 \leq$ $d \leq k-1)$. Suppose that $T\left(r, A_{d}\right) \sim \log M\left(r, A_{d}\right)$ as $r \rightarrow \infty$ outside a set of $r$ of finite logarithmic measure, then one has

(i) if $\sigma_{p}(F)<\sigma_{p}\left(A_{d}\right)$, then every transcendental solution $f(z)$ of (13) satisfies $\sigma_{p+1}(f)=\sigma_{p}\left(A_{d}\right)$; furthermore, if $F(z) \not \equiv 0$, then every transcendental solution $f(z)$ of (13) satisfies $\bar{\lambda}_{p+1}(f)=\lambda_{p+1}(f)=\sigma_{p+1}(f)=\sigma_{p}\left(A_{d}\right)$;

(ii) if $\sigma_{p}(F)>\sigma_{p}\left(A_{d}\right)$ and $\sigma_{p+1}(F) \leq \sigma_{p}\left(A_{d}\right)$, then all solutions of (13) satisfy $\sigma_{p}(f) \geq \sigma_{p}(F)$ and $\sigma_{p+1}(f) \leq$ $\sigma_{p}\left(A_{d}\right)$;

(iii) if $\sigma_{p+1}(F)>\sigma_{p}\left(A_{d}\right)$, then all solutions of (13) satisfy $\sigma_{p+1}(f)=\sigma_{p+1}(F)$, and $\bar{\lambda}_{p+1}(f)=\lambda_{p+1}(f)=$ $\sigma_{p+1}(F)$ holds for all solutions of (13) with at most one exceptional solution $f_{0}$ satisfying $\lambda_{p+1}\left(f_{0}\right)<\sigma_{p+1}(F)$;

(iv) if $\mu_{p}\left(A_{d}\right)=\sigma_{p}\left(A_{d}\right)=\sigma$ and $F(z) \not \equiv 0$ and $\sigma_{p}(F)<\sigma$, then every transcendental solution $f(z)$ of (13) satisfies $\bar{\lambda}_{p+1}(f)=\underline{\lambda}_{p+1}(f)=\bar{\lambda}_{p+1}(f)=\lambda_{p+1}(f)=\sigma$.

Remark 12. Theorem 10 implies that all the solutions of (13) are of regular growth if $A_{d}$ is of regular growth under some conditions; Theorem 11 is an improvement of the Theorem in [14, page 2694] and Theorems 1-2 in [16, page 624]. In fact, by Lemma 15, the gap condition (15) in Theorem 8 implies that $T\left(r, A_{d}\right) \sim \log M\left(r, A_{d}\right)$ as $r \rightarrow \infty$ outside a set of $r$ of finite logarithmic measure; therefore, Theorem 11 is a generalization of Theorem 8 in a sense, but the condition on $A_{d}$ in Theorem 8 is more stringent than that in Theorem 11. In addition, Theorems 8-11 may have polynomial solutions of degree $<d$ if $d>1$.

\section{Preliminary Lemmas}

Lemma 13 (see [17]). Let $f(z)$ be a transcendental meromorphic function, and let $\alpha>1$ be a given constant, for any given constant and for any given $\varepsilon>0$,

(i) there exist a constant $B>0$ and a set $E_{1} \subset(0,+\infty)$ having finite logarithmic measure such that for all $z$ satisfying $|z|=r \notin E_{1}$, one has

$$
\begin{aligned}
& \left|\frac{f^{(j)}(z)}{f^{(i)}(z)}\right| \\
& \quad \leq B\left[\frac{T(\alpha r, f)}{r}(\log r)^{\alpha} \log T(\alpha r, f)\right]^{j-i} \quad(0 \leq i<j) .
\end{aligned}
$$

(ii) There exists a set $H_{1} \subset[0,2 \pi)$ that has linear measure zero a constant $B>0$ that depends only on $\alpha$, for any $\theta \in[0,2 \pi) \backslash H_{1}$, there exists a constant $R_{0}=R_{0}(\theta)>1$ such that for all $z$ satisfying $\arg z=\theta$ and $|z|=r>R_{0}$, one has

$$
\begin{aligned}
& \left|\frac{f^{(j)}(z)}{f^{(i)}(z)}\right| \\
& \quad \leq B[T(\alpha r, f) \log T(\alpha r, f)]^{j-i} \quad(0 \leq i<j) .
\end{aligned}
$$

Remark 14. Throughout this paper, we use $E_{1} \subset(0, \infty)$ to denote a set having finite logarithmic measure or finite linear measure, not always the same at each occurrence. 
Lemma 15 (see [18]). Let $f(z)=\sum_{n=0}^{\infty} c_{\lambda_{n}} z^{\lambda_{n}}$ be an entire function and the sequence of exponents $\left\{\lambda_{n}\right\}$ satisfies the gap condition (15). Then for any given $\varepsilon>0$,

$$
\log L(r, f)>(1-\varepsilon) \log M(r, f)
$$

holds outside a set of finite logarithmic measure, where $M(r, f)=\sup _{|z|=r}|f(z)|, L(r, f)=\inf _{|z|=r}|f(z)|$.

Lemma 16 (see [4]). Let $f(z)$ be an entire function of finite iterated order satisfying $0<\sigma_{p}(f)=\sigma<\infty$ and $\tau_{p}(f)=\tau>$ 0 , then for any given $\beta<\tau$, there exists a set $E_{2} \subset(0,+\infty)$ having infinite logarithmic measure such that for all $r \in E_{2}$, one has

$$
M(r, f)>\exp _{p}\left\{\beta r^{\sigma}\right\}
$$

Lemma 17. Let $f(z)=\sum_{n=0}^{\infty} c_{\lambda_{n}} z^{\lambda_{n}}$ be an entire function of finite iterated order satisfying $0<\sigma_{p}(f)=\sigma<\infty$ and $\tau_{p}(f)=$ $\tau>0$ such that the sequence of exponents $\left\{\lambda_{n}\right\}$ satisfies the gap condition (15). Then, for any given $\beta>\tau$, there exists a set $E_{3} \subset(0,+\infty)$ having infinite logarithmic measure such that for all $|z|=r \in E_{3}$, one has

$$
|f(z)|>\exp _{p}\left\{\beta r^{\sigma}\right\} .
$$

Proof. By Lemma 15, for any given $\varepsilon>0$, we have

$$
|f(z)| \geq L(r, f)>M(r, f)^{(1-\varepsilon)}, \quad\left(r \notin E_{1}\right) .
$$

For any given $\beta<\tau$, we can choose $\beta_{1}$ and sufficiently small $\varepsilon>0$ such that $\beta<\beta_{1}<\tau$ and $\beta<(1-\varepsilon) \beta_{1}<\tau$; by Lemma 16 , there exists a set $E_{2} \subset(0,+\infty)$ having infinite logarithmic measure such that for all $|z|=r \in E_{2} \backslash E_{1}$, we have

$$
\begin{aligned}
|f(z)| & >M(r, f)^{(1-\varepsilon)} \\
& >\left(\exp _{p}\left\{\beta_{1} r^{\sigma}\right\}\right)^{(1-\varepsilon)}>\exp _{p}\left\{\beta r^{\sigma}\right\},
\end{aligned}
$$

where $E_{3}=E_{2} \backslash E_{1}$ is a set having infinite logarithmic measure.

Lemma 18 (see [13]). Let $f(z)$ be a transcendental entire function. Then, there is a set $E_{1} \subset(0,+\infty)$ having finite logarithmic measure such that for all $z$ satisfying $|z|=r \notin E_{1}$ and $|f(z)|=M(r, f)$, one has

$$
\left|\frac{f(z)}{f^{(j)}(z)}\right| \leq 2 r^{j}, \quad(j \in \mathbb{N}) .
$$

Lemma 19 (see $[7,9,10]$ ). Let $A_{0}(z), \ldots, A_{k-1}(z), F(z)$ \# 0 be meromorphic functions, and let $f(z)$ be a meromorphic solution of (13) satisfying one of the following conditions:

(i) $\max \left\{i(F)=q, i\left(A_{j}\right)(j=0, \ldots, k-1)\right\}<i(f)=p+$ $1(p, q \in \mathbb{N})$;

(ii) $b=\max \left\{\sigma_{p+1}(F), \sigma_{p+1}\left(A_{j}\right)(j=0, \ldots, k-1)\right\}<$ $\sigma_{p+1}(f)$

then $\bar{\lambda}_{p+1}(f)=\lambda_{p+1}(f)=\sigma_{p+1}(f)$.
Lemma 20 (see [8]). Let $A_{0}(z), \ldots, A_{k-1}(z), F(z)$ be entire functions of finite iterated order, if $i\left(A_{j}\right) \leq p, i(F) \leq$ $p(j=0, \ldots, k-1)$. Then $\sigma_{p+1}(f) \leq \max \left\{\sigma_{p}\left(A_{j}\right), \sigma_{p}(F), j=\right.$ $0, \ldots, k-1\}$ holds for all solutions of (13).

Lemma 21 (see [2]). Let $g:(0,+\infty) \rightarrow R, h:(0,+\infty) \rightarrow R$ be monotone increasing functions such that

(i) $g(r) \leq h(r)$ outside of an exceptional set of finite linear measure. Then, for any $\alpha>1$, there exists $r_{0}>0$ such that $g(r) \leq h(\alpha r)$ for all $r>r_{0}$.

(ii) $g(r) \leq h(r)$ outside of an exceptional set of finite logarithmic measure. Then, for any $\alpha>1$, there exists $r_{0}>0$ such that $g(r) \leq h\left(r^{\alpha}\right)$ for all $r>r_{0}$.

Lemma 22 (see [19]). Let $f(z)$ be an entire function of finite iterated order satisfying $\mu_{p}(f)=\mu<\infty$. Then, for any given $\varepsilon>0$, there exists a set $E_{4} \subset(0,+\infty)$ having infinite logarithmic measure such that for all $r \in E_{4}$, one has

$$
M(r, f)<\exp _{p}\left\{r^{\mu+\varepsilon}\right\} .
$$

Lemma 23 (see $[2,20]$ ). Let $f(z)$ be a transcendental entire function, let $0<\eta_{1}<1 / 4$ and $z_{r}$ a point such that $\left|z_{r}\right|=r$ and that $\left|f\left(z_{r}\right)\right|>M(r, f) v_{f}(r)^{-(1 / 4)+\eta_{1}}$ holds. Then, there exists a set $E_{1} \subset(0,+\infty)$ of finite logarithmic measure such that

$$
\frac{f^{(j)}\left(z_{r}\right)}{f\left(z_{r}\right)}=\left(\frac{\nu_{f}(r)}{z_{r}}\right)^{j}(1+o(1))
$$

holds for all $j \in \mathbb{N}$ and all $r \notin E_{1}$, where $v_{f}(r)$ is the central index of $f(z)$.

Lemma 24 (see $[7,9]$ ). Let $f(z)$ be an entire function of finite iterated order satisfying $\sigma_{p}(f)=\sigma, \mu_{q}(f)=\mu, p, q \in \mathbb{N}$. Then, one has

$$
\begin{gathered}
\varlimsup_{r \rightarrow \infty} \frac{\log _{p} \nu_{f}(r)}{\log r}=\sigma, \\
\lim _{r \rightarrow \infty} \frac{\log _{q} \nu_{f}(r)}{\log r}=\mu .
\end{gathered}
$$

Lemma 25. Let $A_{j}(z)(j=0, \ldots, k-1), F(z)$ be entire functions of finite iterated order satisfying $\max \left\{\sigma_{p}\left(A_{j}\right)\right.$, $\left.j \neq d, \sigma_{p}(F)\right\} \leq \mu_{p}\left(A_{d}\right)<\infty$. Then, every solution $f(z)$ of (13) satisfies $\mu_{p+1}(f) \leq \mu_{p}\left(A_{d}\right)$.

Proof. By (13), we have

$$
\begin{aligned}
\left|\frac{f^{(k)}}{f}\right| \leq & \left|A_{k-1} \frac{f^{(k-1)}}{f}\right| \\
& +\cdots+\left|A_{1} \frac{f^{\prime}}{f}\right|+\left|A_{0}\right|+\left|\frac{F(z)}{f(z)}\right| .
\end{aligned}
$$

By Lemma 23, there exists a set $E_{1}$ having finite logarithmic measure such that for all $|z|=r \notin E_{1}$ and $|f(z)|=M(r, f)$, we have

$$
\frac{f^{(j)}(z)}{f(z)}=\left(\frac{\nu_{f}(r)}{z}\right)^{j}(1+o(1)), \quad(j \in \mathbb{N}) .
$$


By Lemma 22, for any given $\varepsilon>0$, there exists a set $E_{4}$ having infinite logarithmic measure such that for all $|z|=r \in E_{4}$ and $|f(z)|=M(r, f)>1$, we have

$$
\begin{aligned}
\left|A_{d}(z)\right| & \leq \exp _{p}\left\{r^{\mu_{p}\left(A_{d}\right)+\varepsilon}\right\}, \\
\left|A_{j}(z)\right| & \leq \exp _{p}\left\{r^{\mu_{p}\left(A_{d}\right)+\varepsilon}\right\}, \\
\left|\frac{F(z)}{f(z)}\right| & \leq|F(z)|<\exp _{p}\left\{r^{\mu_{p}\left(A_{d}\right)+\varepsilon}\right\} .
\end{aligned}
$$

Hence from (27)-(29), for any given $\varepsilon>0$ and for all $z$ satisfying $|z|=r \in E_{4} \backslash E_{1}$ and $|f(z)|=M(r, f)$, we have

$$
\begin{aligned}
& \left(\frac{v_{f}(r)}{r}\right)^{k}(1+o(1)) \\
& \quad \leq(k+1)\left(\frac{v_{f}(r)}{r}\right)^{k-1}(1+o(1)) \exp _{p}\left\{r^{\mu_{p}\left(A_{d}\right)+\varepsilon}\right\} .
\end{aligned}
$$

By (30) and Lemma 24, we have $\mu_{p+1}(f) \leq \mu_{p}\left(A_{d}\right)$. Therefore, we complete the proof of Lemma 25.

Lemma 26. Let $A_{0}, A_{1}, \ldots, A_{k-1}, F \neq \equiv 0$ be meromorphic functions of finite iterated order; if $f$ is a meromorphic solution of the (13) and satisfies $b=\max \left\{\sigma_{p+1}(F), \sigma_{p+1}\left(A_{j}\right), j=\right.$ $0, \ldots, k-1\}<\mu_{p+1}(f)$, then $\underline{\bar{\lambda}}_{p+1}(f)=\underline{\lambda}_{p+1}(f)=\mu_{p+1}(f)$.

Proof. By (13), we have

$$
\frac{1}{f}=\frac{1}{F}\left(\frac{f^{(k)}}{f}+A_{k-1} \frac{f^{(k-1)}}{f}+\cdots+A_{0}\right) .
$$

By (31), we get

$$
N\left(r, \frac{1}{f}\right) \leq k \bar{N}\left(r, \frac{1}{f}\right)+N\left(r, \frac{1}{F}\right)+\sum_{j=0}^{k-1} N\left(r, A_{j}\right) .
$$

By the lemma of logarithmic derivative and (31), we have

$$
\begin{aligned}
m\left(r, \frac{1}{f}\right) \leq & m\left(r, \frac{1}{F}\right)+\sum_{j=0}^{k-1} m\left(r, A_{j}\right) \\
& +O\{\log (r T(r, f))\} \quad\left(r \notin E_{1}\right) .
\end{aligned}
$$

By (32) and (33), we have

$$
\begin{aligned}
T(r, f)= & T\left(r, \frac{1}{f}\right)+O(1) \\
\leq & k \bar{N}\left(r, \frac{1}{f}\right)+T\left(r, \frac{1}{F}\right) \\
& +\sum_{j=0}^{k-1} T\left(r, A_{j}\right)+O\{\log (r T(r, f))\} \\
= & k \bar{N}\left(r, \frac{1}{f}\right)+T(r, F)+\sum_{j=0}^{k-1} T\left(r, A_{j}\right) \\
& +O\{\log (r T(r, f))\}, \quad\left(r \notin E_{1}\right) .
\end{aligned}
$$

Since $\max \left\{\sigma_{p+1}(F), \sigma_{p+1}\left(A_{j}\right), j=0,1, \ldots, k-1\right\}<\mu_{p+1}(f)$, for sufficiently large $r$, we have

$$
\begin{aligned}
& T(r, F)=o\{T(r, f)\}, \\
& T\left(r, A_{j}\right)=o\{T(r, f)\}, \quad j=0, \ldots, k-1, \\
& \log (r T(r, f))=o\{T(r, f)\} .
\end{aligned}
$$

By (34)-(35), we have

$$
(1-o(1)) T(r, f) \leq k \bar{N}\left(r, \frac{1}{f}\right), \quad\left(r \notin E_{1}\right) .
$$

By Lemma 21 (i) and by (36), we have $\underline{\bar{\lambda}}_{p+1}(f)=\underline{\lambda}_{p+1}(f)=$ $\mu_{p+1}(f)$.

Lemma 27. Let $f(z)$ be a transcendental entire function, for each sufficiently large $|z|=r$, and let $z_{r}=r e^{i \theta_{r}}$ be a point satisfying $\left|f\left(z_{r}\right)\right|=M(r, f)$. Then, there exists a constant $\delta_{r}$ (> $0)$ such that for all $z$ satisfying $|z|=r \notin E_{1}$ and $\arg z=\theta \in$ $\left[\theta_{r}-\delta_{r}, \theta_{r}+\delta_{r}\right]$, one has

$$
\frac{f^{(j)}(z)}{f(z)}=\left(\frac{\nu_{f}(r)}{z}\right)^{j}(1+o(1)) \quad(j \in \mathbb{N}) .
$$

Proof. If $z_{r}=r e^{i \theta_{r}}$ is a point satisfying $\left|f\left(z_{r}\right)\right|=M(r, f)$, since $|f(z)|$ is continuous in $|z|=r$, then there exists a constant $\delta_{r}(>0)$ such that for all $z$ satisfying $|z|=r$ (large enough) and $\arg z=\theta \in\left[\theta_{r}-\delta_{r}, \theta_{r}+\delta_{r}\right]$, we have

$$
\begin{aligned}
& || f(z)|-| f\left(z_{r}\right)||<\varepsilon ; \\
& \text { that is, }|f(z)|>\frac{1}{2}\left|f\left(z_{r}\right)\right| \\
& =\frac{1}{2} M(r, f) \\
& >M(r, f) v_{f}(r)^{-(1 / 4)+\eta_{1}} \text {. }
\end{aligned}
$$

By Lemma 23, we have

$$
\frac{f^{(j)}(z)}{f(z)}=\left(\frac{\nu_{f}(r)}{z}\right)^{j}(1+o(1)), \quad(j \in \mathbb{N})
$$

holds for all $z$ satisfying $|z|=r \notin E_{1}$ and $\arg z=\theta \in\left[\theta_{r}-\right.$ $\left.\delta_{r}, \theta_{r}+\delta_{r}\right]$.

Lemma 28. Let $f(z)$ be a transcendental entire function, for each sufficiently large $|z|=r$, and let $z_{r}=r e^{i \theta_{r}}$ be a point satisfying $\left|f\left(z_{r}\right)\right|=M(r, f)$. Then, there exists a constant $\delta_{r}$ (> $0)$ such that for all $z$ satisfying $|z|=r \notin E_{1}$ and $\arg z=\theta \in$ $\left[\theta_{r}-\delta_{r}, \theta_{r}+\delta_{r}\right]$, one has

$$
\left|\frac{f(z)}{f^{(j)}(z)}\right| \leq 2 r^{j} \quad(j \in \mathbb{N}) .
$$

Proof. If $z_{r}=r e^{i \theta_{r}}$ is a point satisfying $\left|f\left(z_{r}\right)\right|=M(r, f)$, then by Lemma 27 there exists a constant $\delta_{r}(>0)$ such that for all 
$z$ satisfying $|z|=r \notin E_{1}$ and $\arg z=\theta \in\left[\theta_{r}-\delta_{r}, \theta_{r}+\delta_{r}\right]$, we have

$$
\frac{f^{(j)}(z)}{f(z)}=\left(\frac{\nu_{f}(r)}{z}\right)^{j}(1+o(1)) .
$$

Since $f(z)$ is transcendental, we have $\nu_{f}(r) \rightarrow \infty(r \rightarrow \infty)$. Hence by (41), for all $z$ satisfying $|z|=r \notin E_{1}$ and $\arg z=\theta \in$ $\left[\theta_{r}-\delta_{r}, \theta_{r}+\delta_{r}\right]$, we have

$$
\begin{aligned}
& \left|\frac{f^{(j)}(z)}{f(z)}\right| \geq \frac{1}{2} r^{-j} \\
& \text { that is, }\left|\frac{f(z)}{f^{(j)}(z)}\right| \leq 2 r^{j} \quad(j \in \mathbb{N}) .
\end{aligned}
$$

Therefore, we complete the proof of Lemma 28.

Lemma 29. Let $f(z)$ be an entire function of order $0<$ $\sigma_{p}(f)=\sigma<\infty$. Then for any given $\varepsilon>0$, there exists a set $E_{5} \subset(0,+\infty)$ with positive upper logarithmic density such that for all $|z|=r \in E_{5}$, one has

$$
M(r, f)>\exp _{p}\left\{r^{\sigma-\varepsilon}\right\} .
$$

Proof. Since $0<\sigma_{p}(f)=\sigma<\infty$, then for any given $\varepsilon>0$, there exists an increasing sequence $\left\{r_{n}\right\}$ tending to $\infty$ such that for $n \geq n_{4}$, we have

$$
M\left(r_{n}, f\right)>\exp _{p}\left\{r_{n}^{\sigma-(\varepsilon / 2)}\right\} .
$$

Since $\varepsilon>0$, we can choose $\alpha$ to satisfy $1<\alpha<(\sigma-(\varepsilon / 2)) /(\sigma-$ $\varepsilon)$. Then for all $r \in\left[r_{n}, r_{n}^{\alpha}\right]\left(n \geq n_{4}\right)$, we have

$$
\begin{aligned}
M(r, f) & \geq M\left(r_{n}, f\right)>\exp _{p}\left\{r_{n}^{\sigma-(\varepsilon / 2)}\right\} \\
& \geq \exp _{p}\left\{r^{(\sigma-(\varepsilon / 2)) / \alpha}\right\}>\exp _{p}\left\{r^{\sigma-\varepsilon}\right\} .
\end{aligned}
$$

Setting $E_{5}=\bigcup_{n=n_{4}}^{\infty}\left[r_{n}, r_{n}^{\alpha}\right]$, we have

$$
\begin{aligned}
\overline{\log \text { dens }} E_{5} & =\varlimsup_{r \rightarrow \infty} \frac{m_{l}\left(E_{5} \cap[1, r]\right)}{\log r} \\
& \geq \varlimsup_{n \rightarrow \infty} \frac{m_{l}\left(E_{5} \cap\left[1, r_{n}^{\alpha}\right]\right)}{\log r_{n}^{\alpha}} \\
& \geq \lim _{n \rightarrow \infty} \frac{m_{l}\left(\left[r_{n}, r_{n}^{\alpha}\right]\right)}{\log r_{n}^{\alpha}}=\frac{\alpha-1}{\alpha}>0 .
\end{aligned}
$$

Thus, Lemma 29 is proved.

Lemma 30. Let $f(z)$ be a transcendental entire function satisfying $0<\sigma_{p}(f)=\sigma<\infty$ and $T(r, f) \sim \log M(r, f)$ as $r \rightarrow \infty$ outside a set $r$ of finite logarithmic measure. Then for any given $\varepsilon>0$, there exists a set $E_{6} \subset(0,+\infty)$ with positive upper logarithmic density and a set $H_{2} \subset[0,2 \pi)$ with linear measure zero such that for all $z$ satisfying $r \in E_{6}$ and $\arg z=\theta \in[0,2 \pi) \backslash H_{2}$, one has

$$
\left|f\left(r e^{i \theta}\right)\right|>\exp _{p}\left\{r^{\sigma-\varepsilon}\right\} .
$$

Proof. Since $m(r, f) \sim \log M(r, f)$ as $r \rightarrow \infty\left(r \notin E_{1}\right)$, then by the definition of $m(r, f)$, there exists a set $H_{2} \subset$ $[0,2 \pi)$ having linear measure zero such that for all $z$ satisfying $\arg z=\theta \in[0,2 \pi) \backslash H_{2}$, we have

$$
\left|f\left(r e^{i \theta}\right)\right|>M(r, f)^{1-\varepsilon} \quad\left(r \notin E_{1}\right) .
$$

By Lemma 29, for any given $\varepsilon>0$, there exists a set $E_{6} \subset$ $(0,+\infty)$ with positive upper logarithmic density, we have

$$
M(r, f)>\exp _{p}\left\{r^{\sigma-(\varepsilon / 2)}\right\} .
$$

By (48) and (49), for any given $\varepsilon>0$ and for all $z$ satisfying $|z|=r \in E_{6} \backslash E_{1}$ and $\arg z=\theta \in[0,2 \pi) \backslash H_{2}$, we have

$$
\left|f\left(r e^{i \theta}\right)\right|>M(r, f)^{1-\varepsilon}>\left(\exp _{p}\left\{r^{\sigma-(\varepsilon / 2)}\right\}\right)^{1-\varepsilon}>\exp _{p}\left\{r^{\sigma-\varepsilon}\right\} .
$$

Therefore, we complete the proof of Lemma 30.

\section{Proofs of Theorems 8-11}

Proof of Theorem 8. (i) Assume that $f(z)$ is a transcendental solution of (13). By (13), we have

$$
\begin{aligned}
\left|A_{d}\right| \leq & \left|\frac{f^{(k)}}{f^{(d)}}\right|+\cdots+\left|A_{d+1}\right|\left|\frac{f^{(d+1)}}{f^{(d)}}\right| \\
& +\left|\frac{f}{f^{(d)}}\right|\left(\left|A_{d-1}\right|\left|\frac{f^{(d-1)}}{f}\right|+\cdots+\left|A_{0}\right|+\left|\frac{F}{f}\right|\right) .
\end{aligned}
$$

By Lemma 13 (i), there exists a set $E_{1} \subset[1, \infty)$ having finite logarithmic measure and a constant $B>0$ such that

$$
\left|\frac{f^{(j)}(z)}{f^{(i)}(z)}\right| \leq B(T(2 r, f))^{2 k}, \quad(0 \leq i<j \leq k)
$$

holds for all $|z|=r \notin E_{1}$ and for sufficiently large $r$. Since $\max \left\{\sigma_{p}\left(A_{j}\right), j \neq d, \sigma_{p}(F)\right\} \leq \sigma_{p}\left(A_{d}\right)$ and $\max \left\{\tau_{p}\left(A_{j}\right), \tau_{p}(F):\right.$ $\left.\sigma_{p}\left(A_{j}\right)=\sigma_{p}\left(A_{d}\right)=\sigma_{p}(F)\right\}<\tau_{p}\left(A_{d}\right)$, we choose $\alpha_{1}, \beta_{1}$ to satisfy $\max \left\{\tau_{p}\left(A_{j}\right), j \neq d, \tau_{p}(F)\right\}<\alpha_{1}<\beta_{1}<\tau_{p}\left(A_{d}\right)$; by Lemma 17, there exists a set $E_{3} \subset(0,+\infty)$ having infinite logarithmic measure such that for all $z$ satisfying $|z|=r \in E_{3}$ and for sufficiently large $r$, we have

$$
\begin{aligned}
& \left|A_{d}(z)\right|>\exp _{p}\left\{\beta_{1} r^{\sigma_{p}\left(A_{d}\right)}\right\}, \\
& \left|A_{j}(z)\right|<\exp _{p}\left\{\alpha_{1} r^{\sigma_{p}\left(A_{d}\right)}\right\}, \quad j \neq d .
\end{aligned}
$$

By Lemma 18, there exists a set $E_{1} \subset(0,+\infty)$ having finite logarithmic measure such that for all $z$ satisfying $|f(z)|=$ $M(r, f)>1$ and $|z|=r \notin E_{1}$, we have

$$
\begin{aligned}
\left|\frac{f(z)}{f^{(d)}(z)}\right| & \leq 2 r^{d}, \\
\left|\frac{F(z)}{f(z)}\right| & \leq|F(z)|<\exp _{p}\left\{\alpha_{1} r^{\sigma_{p}\left(A_{d}\right)}\right\} .
\end{aligned}
$$


Hence from (51)-(54), for all $z$ satisfying $|z|=r \in E_{3} \backslash E_{1}$ and $|f(z)|=M(r, f)$, we have

$$
\begin{aligned}
\exp _{p} & \left\{\beta_{1} r^{\sigma_{p}\left(A_{d}\right)}\right\} \\
\leq & 2 B(k+1) r^{d} \exp \left\{\alpha_{1} r^{\sigma_{p}\left(A_{d}\right)}\right\}[T(2 r, f)]^{2 k} .
\end{aligned}
$$

By (55), we have

$$
\sigma_{p+1}(f)=\varlimsup_{r \rightarrow \infty} \frac{\log _{p+1} T(r, f)}{\log r} \geq \sigma_{p}\left(A_{d}\right) .
$$

On the other hand, by Lemma 20, we have $\sigma_{p+1}(f) \leq \sigma_{p}\left(A_{d}\right)$. Therefore, every transcendental solution $f(z)$ of (13) satisfies $\sigma_{p+1}(f)=\sigma_{p}\left(A_{d}\right)$. Furthermore if $F(z) \neq \equiv$, then by Lemma 19, we have that every transcendental solution $f(z)$ of (13) satisfies $\bar{\lambda}_{p+1}(f)=\lambda_{p+1}(f)=\sigma_{p+1}(f)=\sigma_{p}\left(A_{d}\right)$.

(ii) We assume that $f$ is a solution of (13). By the elementary theory of differential equations, all the solutions of (13) are entire functions and have the form

$$
f=f^{*}+C_{1} f_{1}+C_{2} f_{2}+\cdots+C_{k} f_{k},
$$

where $C_{1}, \ldots, C_{k}$ are complex constants, $\left\{f_{1}, \ldots, f_{k}\right\}$ is a solution base of (12), and $f^{*}$ is a solution of (13) and has the form

$$
f^{*}=D_{1} f_{1}+D_{2} f_{2}+\cdots+D_{k} f_{k}
$$

where $D_{1}, \ldots, D_{k}$ are certain entire functions satisfying

$$
D_{j}^{\prime}=F \cdot G_{j}\left(f_{1}, \ldots, f_{k}\right) \cdot W\left(f_{1}, \ldots, f_{k}\right)^{-1} \quad(j=1, \ldots, k),
$$

where $G_{j}\left(f_{1}, \ldots, f_{k}\right)$ are differential polynomials in $f_{1}, \ldots, f_{k}$ and their derivative with constant coefficients, and $W\left(f_{1}, \ldots, f_{k}\right)$ is the Wronskian of $f_{1}, \ldots, f_{k}$. By Theorem A, we have $\sigma_{p+1}\left(f_{j}\right) \leq \sigma_{p}\left(A_{d}\right)(j=1,2, \ldots, k)$; then by (57)-(59), we get

$$
\begin{aligned}
\sigma_{p+1}(f) & \leq \max \left\{\sigma_{p+1}\left(f_{j}\right), \sigma_{p+1}(F), j=1, \ldots, k\right\} \\
& \leq \sigma_{p}\left(A_{d}\right) .
\end{aligned}
$$

Since $\sigma_{p}(F)>\sigma_{p}\left(A_{d}\right)$, it is easy to see that $\sigma_{p}(f) \geq \sigma_{p}(F)$ by (13).

(iii) Suppose that $f$ is a solution of (13), it is easy to see that $\sigma_{p+1}(f) \geq \sigma_{p+1}(F)$ by $(13)$. On the other hand, since $\sigma_{p+1}(F)>$ $\sigma_{p}\left(A_{d}\right)$ and by $(57)-(59)$, we have

$$
\begin{aligned}
\sigma_{p+1}(f) & \leq \max \left\{\sigma_{p+1}\left(f_{j}\right), \sigma_{p+1}(F), j=1, \ldots, k\right\} \\
& \leq \sigma_{p+1}(F) .
\end{aligned}
$$

Therefore, all solutions of (13) satisfy $\sigma_{p+1}(f)=\sigma_{p+1}(F)$.

By the same proof in Theorem 4.2 in [8, page 401], we can obtain that all solutions of (13) satisfying $\bar{\lambda}_{p+1}(f)=$ $\lambda_{p+1}(f)=\sigma_{p+1}(F)$ with at most one exceptional solution $f_{0}$ satisfying $\lambda_{p+1}\left(f_{0}\right)<\sigma_{p+1}(F)$.
Proof of Theorem 10. Suppose that $f(z)$ is a transcendental solution of (13), by the same proof in Theorem 8, we have $\sigma_{p+1}(f)=\sigma_{p}\left(A_{d}\right)=\sigma$. Thus, it remains to show that $\mu_{p+1}(f)=\mu_{p}\left(A_{d}\right)=\sigma$. We choose $\alpha_{2}, \beta_{2}$ to satisfy

$$
\max \left\{\sigma_{p}\left(A_{j}\right), j \neq d, \sigma_{p}(F)\right\}<\alpha_{2}<\beta_{2}<\sigma .
$$

Since the sequence of exponents $\left\{\lambda_{n}\right\}$ of $A_{d}$ satisfies (15) and $\mu_{p}\left(A_{d}\right)=\sigma$, then by Lemma 15 , there exists a set $E_{1}$ having finite logarithmic measure such that for all sufficiently large $r \notin E_{1}$, we have

$$
\begin{aligned}
& \left|A_{d}(z)\right| \geq \exp _{p}\left\{r^{\beta_{2}}\right\}, \\
& \left|A_{j}(z)\right| \leq \exp _{p}\left\{r^{\alpha_{2}}\right\}, \quad j \neq d .
\end{aligned}
$$

Hence from (51), (52), (54), and (63), for all $z$ satisfying $|z|=$ $r \notin E_{1}$ and $|f(z)|=M(r, f)$, we have

$$
\exp _{p}\left\{r^{\beta_{2}}\right\} \leq 2 B(k+1) r^{d} \exp _{p}\left\{r^{\alpha_{2}}\right\}[T(2 r, f)]^{2 k} .
$$

Since $\beta_{2}$ is arbitrarily close to $\sigma$, by (64) and Lemma 21 (ii), we have

$$
\mu_{p+1}(f)=\lim _{r \rightarrow \infty} \frac{\log _{p+1} T(r, f)}{\log r} \geq \sigma .
$$

On the other hand, by Lemma 26 , we have $\mu_{p+1}(f) \leq$ $\mu_{p}\left(A_{d}\right)=\sigma$; therefore, every transcendental solution of (13) satisfies $\mu_{p+1}(f)=\sigma$.

Proof of Theorem 11. (i) By Lemma 20, we know that every solution of (13) satisfies $\sigma_{p+1}(f) \leq \sigma_{p}\left(A_{d}\right)$. In the following, we show that every transcendental solution $f(z)$ of (13) satisfies $\sigma_{p+1}(f) \geq \sigma_{p}\left(A_{d}\right)$. Suppose that $f(z)$ is a transcendental solution of (13). For each sufficiently large circle $|z|=r$, we take a point $z_{r}=r e^{i \theta_{r}}$ satisfying $\left|f\left(z_{r}\right)\right|=M(r, f)$. By Lemma 28, there exist a constant $\delta_{r}>0$ and a set $E_{1}$ such that for all $z$ satisfying $|z|=r \notin E_{1}$ and $\arg z=\theta \epsilon$ $\left[\theta_{r}-\delta_{r}, \theta_{r}+\delta_{r}\right]$, we have

$$
\left|\frac{f(z)}{f^{(d)}(z)}\right| \leq 2 r^{d}
$$

By Lemma 13 (ii), there exist a set $H_{1} \subset[0,2 \pi)$ having linear measure zero and a constant $B>0$ such that for sufficiently large $r$ and for all $z$ satisfying $\arg z=\theta \in\left[\theta_{r}-\delta_{r}, \theta_{r}+\delta_{r}\right] \backslash$ $\mathrm{H}_{1}$, we have

$$
\left|\frac{f^{(j)}(z)}{f^{(i)}(z)}\right| \leq B[T(2 r, f)]^{2 k} \quad(0 \leq i<j \leq k) .
$$

Setting $\max \left\{\sigma_{p}\left(A_{j}\right), \sigma_{p}(F), j \neq d\right\}=b<\sigma_{p}\left(A_{d}\right)$, for all $z$ satisfying $|z|=r \notin E_{1}$ and $\arg z=\theta \in\left[\theta_{r}-\delta_{r}, \theta_{r}+\delta_{r}\right]$ and for any given $\varepsilon\left(0<2 \varepsilon<\sigma_{p}\left(A_{d}\right)-b\right)$, we have

$$
\begin{gathered}
\left|A_{j}(z)\right| \leq \exp \left\{r^{b+\varepsilon}\right\} \quad(j \neq d), \\
\left|\frac{F(z)}{f(z)}\right| \leq|F(z)| \leq \exp \left\{r^{b+\varepsilon}\right\} .
\end{gathered}
$$


Since $T\left(r, A_{d}\right) \sim \log M\left(r, A_{d}\right)$ as $r \rightarrow \infty\left(r \notin E_{1}\right)$, by Lemma 30 , for any given $\varepsilon>0$, there exists a set $E_{6} \subset(0, \infty)$ with $\overline{\log \text { dens }} E_{6}>0$ and a set $H_{2} \subset[0,2 \pi)$ with linear measure zero such that for all $z$ satisfying $|z|=r \in E_{6}$ and $\arg z=\theta \in\left[\theta_{r}-\delta_{r}, \theta_{r}+\delta_{r}\right] \backslash H_{2}$, we have

$$
\left|A_{d}(z)\right|>\exp _{p}\left\{r^{\sigma_{p}\left(A_{d}\right)-\varepsilon}\right\} .
$$

Substituting (66)-(69) into (51), for all $z$ satisfying $|z|=r \epsilon$ $E_{6} \backslash E_{1}$ and $\arg z=\theta \in\left[\theta_{r}-\delta_{r}, \theta_{r}+\delta_{r}\right] \backslash\left(H_{1} \cup H_{2}\right)$, we have

$$
\begin{aligned}
& \exp _{p}\left\{r^{\sigma_{p}\left(A_{d}\right)-\varepsilon}\right\} \\
& \quad \leq(k+1) B[T(2 r, f)]^{2 k} \cdot 2 r^{d} \cdot \exp _{p}\left\{r^{b+\varepsilon}\right\} .
\end{aligned}
$$

From (70), we have $\sigma_{p+1}(f) \geq \sigma_{p}\left(A_{d}\right)$. Therefore, every transcendental solution $f(z)$ of (13) satisfies $\sigma_{p+1}(f)=$ $\sigma_{p}\left(A_{d}\right)$. Furthermore, if $F(z) \not \equiv 0$, then every transcendental solution $f(z)$ of (13) satisfies $\bar{\lambda}_{p+1}(f)=\lambda_{p+1}(f)=\sigma_{p+1}(f)=$ $\sigma_{p}\left(A_{d}\right)$.

(ii)-(iv) By the same proof in Theorems 8 and 10, we can obtain the conclusions (ii)-(iv).

\section{Acknowledgments}

This project was supported by the National Natural Science Foundation of China (Grant nos. 11171119, 11261024, and 61202313), the Natural Science Foundation of Jiangxi Province in China (20122BAB211005, 20114BAB211003, 2010GQS0119, and 20122BAB201016), and the Foundation of Education Bureau of Jiangxi Province in China (Grant no. GJJ12206).

\section{References}

[1] W. K. Hayman, Meromorphic Functions, Clarendon Press, Oxford, UK, 1964.

[2] I. Laine, Nevanlinna Theory and Complex Differential Equations, vol. 15, Walter de Gruyter, Berlin, Germany, 1993.

[3] C.-C. Yang and H.-X. Yi, Uniqueness Theory of Meromorphic Functions, vol. 557, Kluwer Academic, Dordrecht, The Netherlands, 2003.

[4] B. Belaïdi, "Growth and oscillation of solutions to linear differential equations with entire coefficients having the same order," Electronic Journal of Differential Equations, no. 70, pp. $1-10,2009$.

[5] B. Belaidi, "On the iterated order and the fixed points of entire solutions of some complex linear differential equations," Electronic Journal of Qualitative Theory of Differential Equations, no. 9, pp. 1-11, 2006.

[6] L. G. Bernal, "On growth $k$-order of solutions of a complex homogeneous linear differential equation," Proceedings of the American Mathematical Society, vol. 101, no. 2, pp. 317-322, 1987.

[7] T. Cao and H. Yi, "On the complex oscillation of higher order linear differential equations with meromorphic coefficients," Journal of Systems Science \& Complexity, vol. 20, no. 1, pp. 135148, 2007.

[8] L. Kinnunen, "Linear differential equations with solutions of finite iterated order," Southeast Asian Bulletin of Mathematics, vol. 22, no. 4, pp. 385-405, 1998.
[9] J. Tu and Z.-X. Chen, "Growth of solutions of complex differential equations with meromorphic coefficients of finite iterated order," Southeast Asian Bulletin of Mathematics, vol. 33, no. 1, pp. 153-164, 2009.

[10] J. Tu and T. Long, "Oscillation of complex high order linear differential equations with coefficients of finite iterated order," Electronic Journal of Qualitative Theory of Differential Equations, no. 66, pp. 1-13, 2009.

[11] Y.-M. Chiang and W. K. Hayman, "Estimates on the growth of meromorphic solutions of linear differential equations," Commentarii Mathematici Helvetici, vol. 79, no. 3, pp. 451-470, 2004.

[12] J. Tu and G. T. Deng, "Growth of solutions to higher-order linear differential equations with the coefficient $A_{0}$ being dominant," Acta Mathematica Scientia A, vol. 30, no. 4, pp. 945-952, 2010.

[13] Z.-X. Chen and C.-C. Yang, "Quantitative estimations on the zeros and growths of entire solutions of linear differential equations," Complex Variables, vol. 42, no. 2, pp. 119-133, 2000.

[14] I. Laine and P. C. Wu, "Growth of solutions of second order linear differential equations," Proceedings of the American Mathematical Society, vol. 128, no. 9, pp. 2693-2703, 2000.

[15] J. Tu and J. Liu, "Growth of solutions of a class of higher order linear differential equations with coefficients being gap series," Journal of Mathematical Research and Exposition, vol. 29, no. 3, pp. 563-567, 2009.

[16] J. Tu and G. Deng, "Growth of solutions of certain higher order linear differential equations," Complex Variables and Elliptic Equations, vol. 53, no. 7, pp. 623-631, 2008.

[17] G. G. Gundersen, "Estimates for the logarithmic derivative of a meromorphic function, plus similar estimates," Journal of the London Mathematical Society, vol. 37, no. 1, pp. 88-104, 1988.

[18] T. Kövari, "A gap-theorem for entire functions of infinite order," The Michigan Mathematical Journal, vol. 12, pp. 133-140, 1965.

[19] H. Hu and X. M. Zheng, "Growth of solutions to linear differential equations with entire coefficients," Electronic Journal of Differential Equations, no. 226, pp. 1-15, 2012.

[20] W. K. Hayman, "The local growth of power series: a survey of the Wiman-Valiron method," Canadian Mathematical Bulletin, vol. 17, no. 3, pp. 317-358, 1974. 


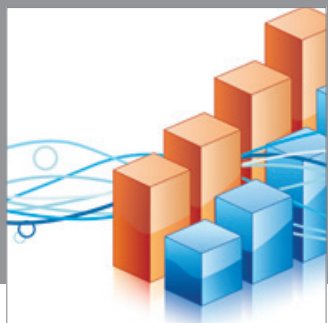

Advances in

Operations Research

mansans

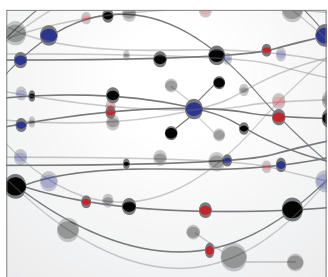

The Scientific World Journal
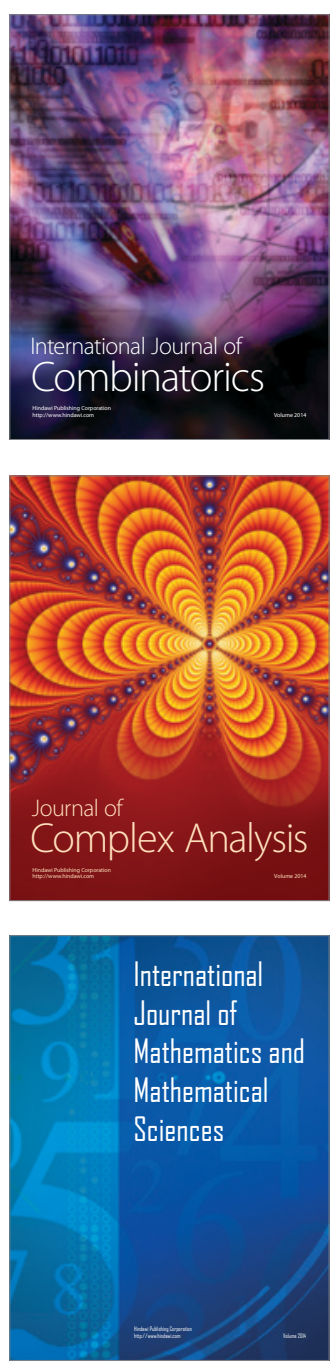
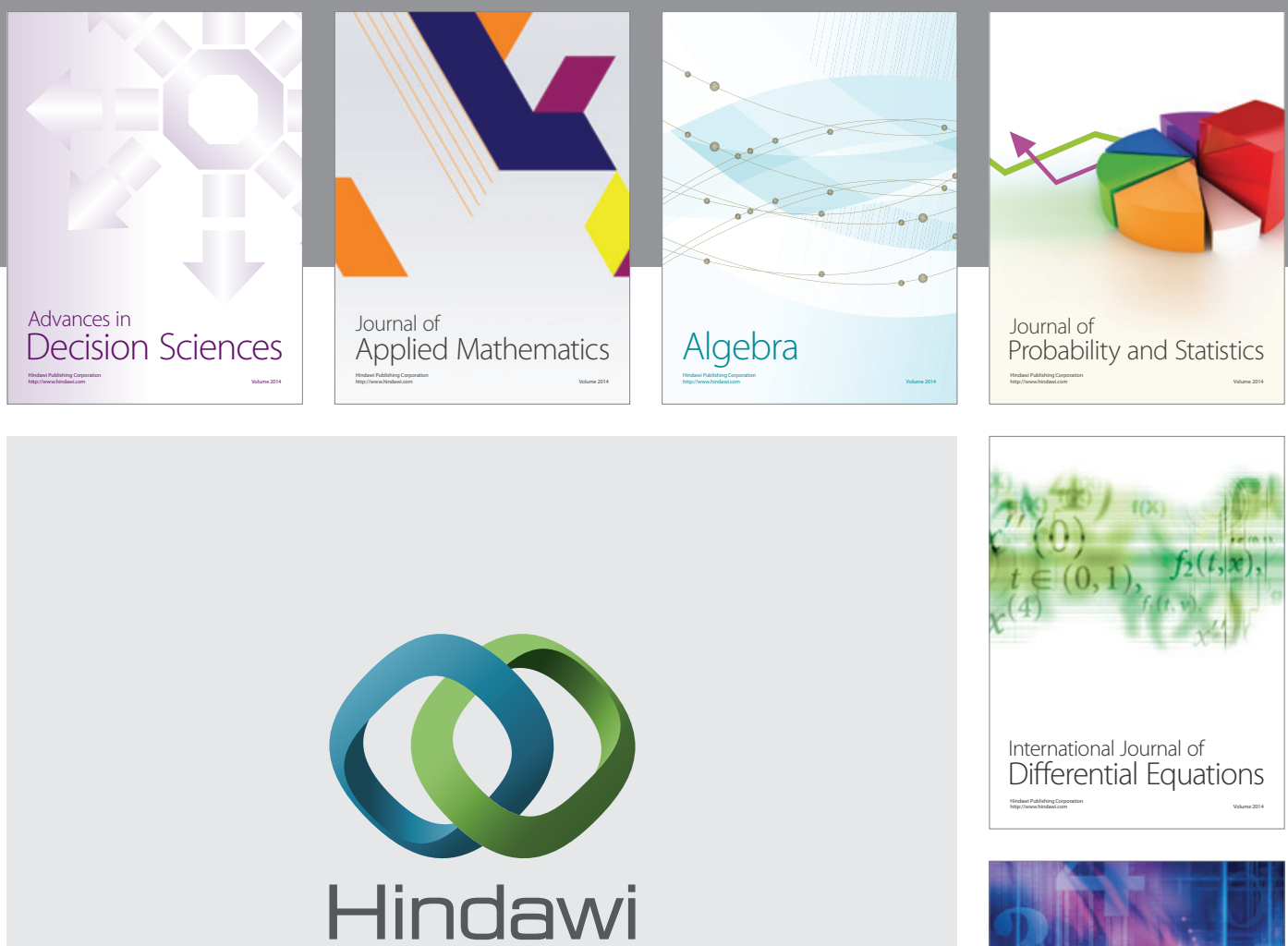

Submit your manuscripts at http://www.hindawi.com
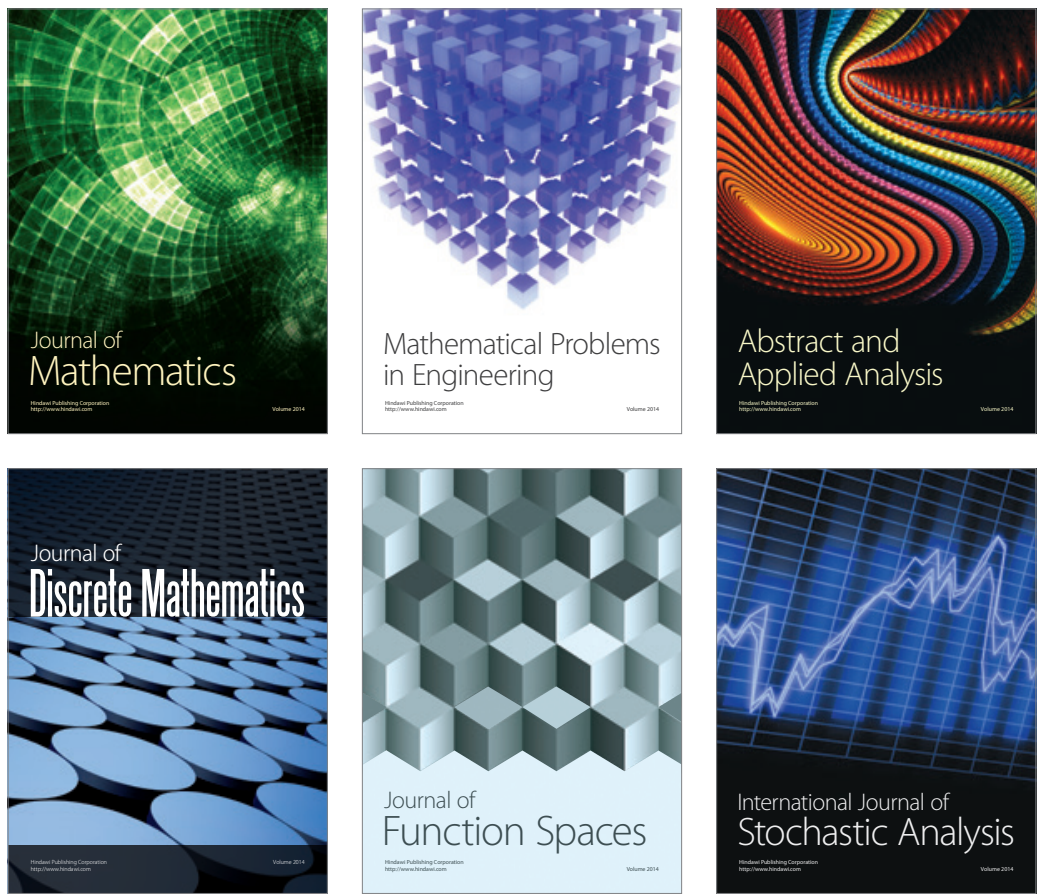

Journal of

Function Spaces

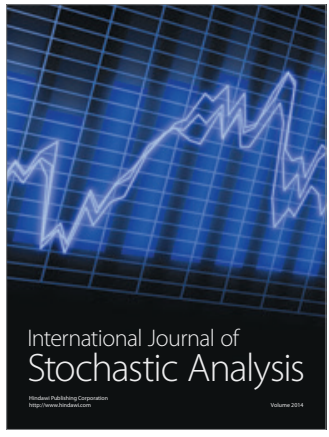

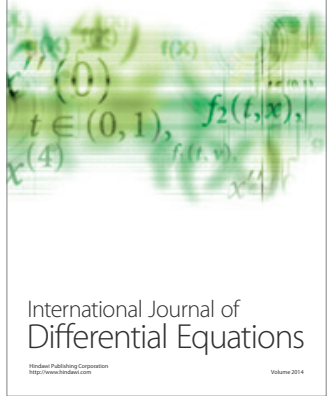
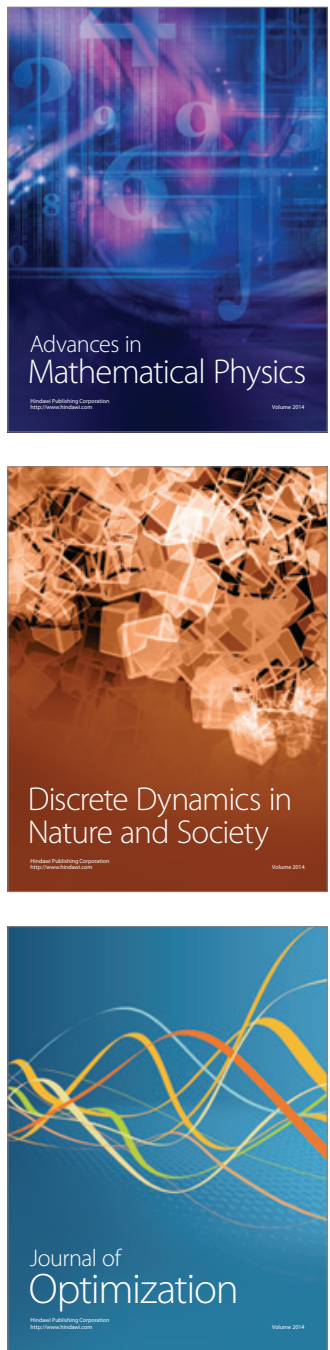